Pacific Journal of Mathematics

A COMBINATORIAL MATRIX IN 3-MANIFOLD THEORY G Ko AND LAWRENCE SMOLINSKY 


\section{A COMBINATORIAL MATRIX IN 3-MANIFOLD THEORY}

\section{Ki Hyoung Ko and Lawrence SMolinsky}

In this paper we study a combinatorial matrix considered by W. B. R. Lickorish. We prove a conjecture by Lickorish that completes his topological and combinatorial proof of the existence of the Witten-Reshetikhin-Turaev 3-manifold invariants. We derive a recursive formula for the determinant of the matrix and discover some interesting numerical relations.

In this paper we study the matrix $A(n)$ which was defined by W. B. R. Lickorish [3]. We prove a result required by Lickorish which completes his topological and combinatorial approach to the 3-manifold invariants of Witten-Reshetikhin-Turaev [4], [5]. This matrix arises from a pairing on a set of geometric configurations. These are the configurations of $n$ nonintersecting arcs in the disk with $2 n$ specified boundary points. There are $C_{n}$ such configurations where $C_{n}$ is the $n$th Catalan number so the matrix increases in size very rapidly. The Catalan numbers were discovered by Euler who considered the ways to partition a polygon into triangles [1]. These two counting problems correspond naturally by considering "restricted sequences".

The matrix has entries in $\mathbf{Z}[\delta]$. Lickorish needed that $\operatorname{det} A(n)=0$ if $\delta= \pm 2 \cos \frac{\pi}{n+1}$. We find a recursive formula for $\operatorname{det} A(n)$ and show that all the roots are of the form $2 \cos \frac{k \pi}{m+1}$ for $1 \leq m \leq n$ and $1 \leq$ $k \leq m$ and verify the result. Using this formula, we derive a simple rule that allows one to recursively compute $\operatorname{det} A(n)$ by generating all of its factors.

There have been three approaches to study polynomial invariants of classical links: the topological and combinatorial approach considered by Kauffman, Lickorish and many other topologists; the study of quantized Yang-Baxter equations and related Lie algebras by Reshetikhin and Turaev; and the study of subfactors and traces of von Neumann and Hecke algebras by Jones. We took a topological and combinatorial viewpoint. The authors have been informed that the essential result needed by Lickorish could have been obtained by pursuing the two other approaches. 
1. Combinatorial manipulation. Let $D_{n}$ be the set of configurations of $n$ non-intersecting arcs on a disk joining $2 n$ points on the boundary of the disk. We draw these configurations by taking $S^{1}$ to be $[0,1] / 0 \sim 1$ as in Fig. 1 .

The cardinality of $D_{n}$ is equal to $(2 n) ! / n !(n+1) !$, known as the Catalan number, denoted here by $C_{n}$. It satisfies the recursive relation:

$$
C_{n}=C_{n-1}+C_{1} C_{n-2}+\cdots+C_{n-2} C_{1}+C_{n-1} \text {. }
$$

We can inductively represent the elements of $D_{n}$ by sequences of $n$ integers $\left(a_{1}, a_{2}, \ldots, a_{n}\right)$ where $1 \leq a_{i} \leq n-i+1$. The first entry $a_{1}$ means that there is an innermost arc in the configuration joining the $a_{1}$ th point and the $\left(a_{1}+1\right)$ th point on the interval. One then deletes that arc and has an element of $D_{n-1}$ remaining. The sequence $\left(a_{2}, a_{3}, \ldots, a_{n}\right)$ then represents this element of $D_{n-1}$. See Fig. 2 for an example.

Note that every configuration in $D_{n}$ must contain an innermost arc between adjacent points among the first $n+1$ points. Thus this representation captures all possible configurations but with repetitions. For example the configuration in Fig. 3 has 12 distinct associated sequences.
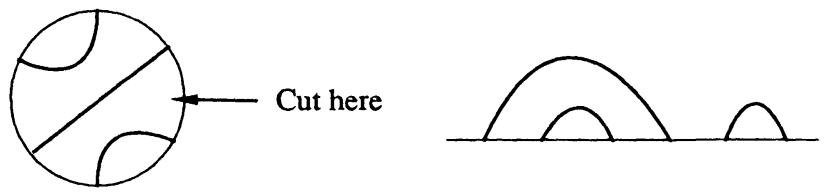

FIGURE 1

$(4,1,2,1)$ represents
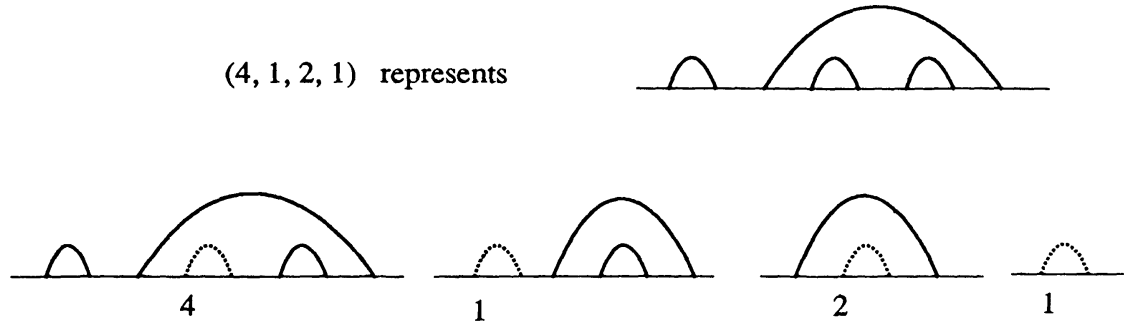

FIGURE 2 


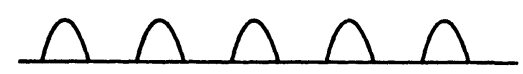

FIGURE 3

Given such a sequence $\left(a_{1}, a_{2}, \ldots, a_{n}\right)$ one may construct the unique configuration inductively. Into the configuration $\left(a_{2}, a_{3}, \ldots\right.$, $\left.a_{n}\right)$ one can insert two additional points between $\left(a_{1}-1\right)$ st and $a_{1}$ th points then joining these two new points by an innermost arc. Thus two distinct configurations cannot have the same sequence. To a given configuration in $D_{n}$, one can associate the unique sequence $\left(a_{1}, a_{2}, \ldots, a_{n}\right)$ in which $a_{1}$ indicates the initial position of the first occurring innermost arc and $a_{2}$ does the same for the configuration without the previous innermost arc and so on. Such a sequence is said to be restricted.

Proposition 1.1. A sequence $\left(a_{1}, a_{2}, \ldots, a_{n}\right)$ of a configuration is restricted if and only if $a_{i-1}-1 \leq a_{i}$ for all $i=2, \ldots, n$.

Proof. For a restricted sequence $\left(a_{1}, a_{2}, \ldots, a_{n}\right)$, it is enough to prove $a_{1}-1 \leq a_{2}$ since $\left(a_{2}, \ldots, a_{n}\right)$ is also a restricted sequence. After removing the first innermost arc, either the second innermost arc or the arc joining the $\left(a_{1}-1\right)$ th and the $\left(a_{1}+2\right)$ th point in the original configuration will become the first innermost arc in the remaining configuration. Thus $a_{1}-1 \leq a_{2}$.

Conversely if $a_{i-1}-1 \leq a_{i}$, then the newly inserted innermost arc into the configuration of $\left(a_{i-1}, \ldots, a_{n}\right)$ becomes the first innermost arc in the configuration of $\left(a_{i}, \ldots, a_{n}\right)$.

REMARK. The number of ways to divide an $(n+2)$ gon into triangles or the number of ways to interpret the product $x_{1} x_{2} \cdots x_{n+1}$ in a non-associative algebra is equal to the Catalan number $C_{n}$. Restricted sequences are useful to see the correspondence between these and configurations defined earlier. Label the vertex of the $(n+2)$ gon counterclockwise 1 through $n$ except fixed adjacent vertices. A triangle in a partition is said to be outermost if it has a vertex contained in no other triangle. To a partition of the $(n+2)$ gon we assign the sequence $\left(a_{1}, a_{2}, \ldots, a_{n}\right)$ where $a_{1}$ is the vertex that is solely contained in the first occurring outermost triangle. Then the sequence $\left(a_{2}, \ldots, a_{n}\right)$ inductively represents the partition of the $(n+1)$ gon obtained by deleting the vertex $a_{1}$ and its adjacent sides. See Fig. 4 for an example. 
$(1,2,2,1)$ is the unique representation of
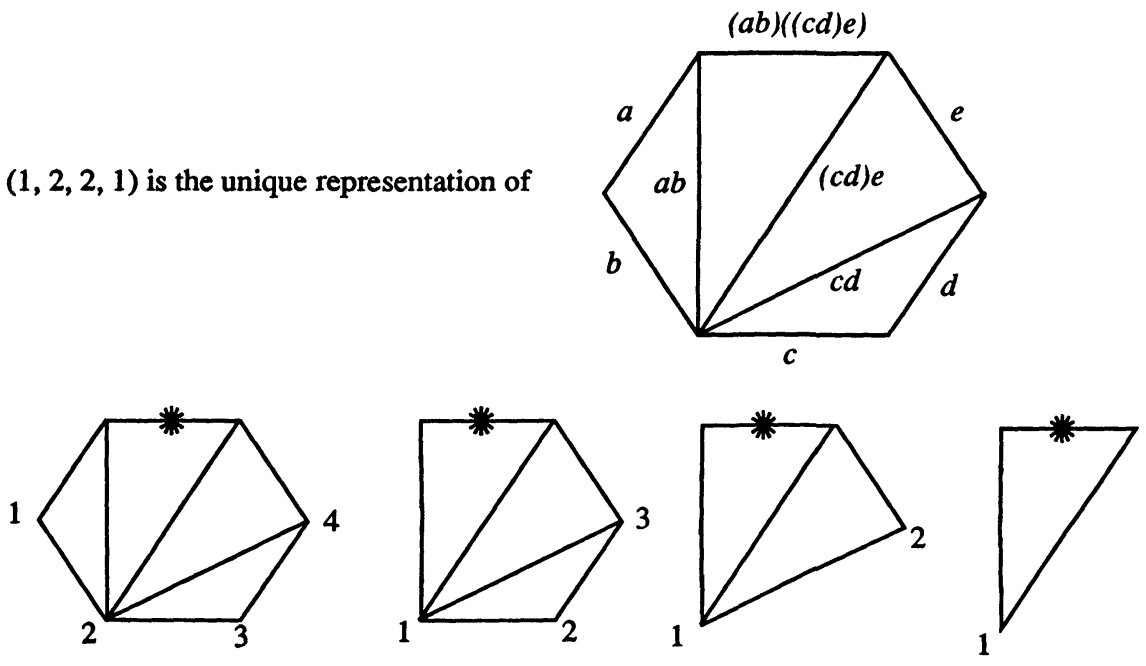

FIGURE 4

We give the lexicographic order to the set of all the sequences of configurations, i.e., $\left(a_{1}, a_{2}, \ldots, a_{n}\right)<\left(b_{1}, b_{2}, \ldots, b_{n}\right)$ if there is an index $k$ such that $a_{1}=b_{1}, \ldots, a_{k-1}=b_{k-1}$ and $a_{k}<b_{k}$. If two distinct sequences $\alpha$ and $\beta$ represent the same configuration and $\alpha$ is restricted, then clearly $\alpha<\beta$.

Let $B(n, k)$ be the set of restricted sequences of length $n$ with initial entry $k$ and let $b(n, k)$ be the cardinality $|B(n, k)|$ of the set $B(n, k)$. Since $D_{n}$ can be identified with the set of all restricted sequences of length $n, C_{n}=\sum_{k=1}^{n} b(n, k)$. It is convenient to set $b(n, k)=0$ for $k=0$ or $k>n$.

Proposition 1.2. $b(n, k)=\sum_{i=k-1}^{n-1} b(n-1, i)$ for $k=1, \ldots, n$.

Proof. Immediately follows from Proposition 1.1.

It is interesting that $b(n, 1)=b(n, 2)=C_{n-1}, b(n, n-1)=n-1$, and $b(n, n)=1$. The only element in $B(n, n)$ is $(n, n-1, \ldots, 2,1)$

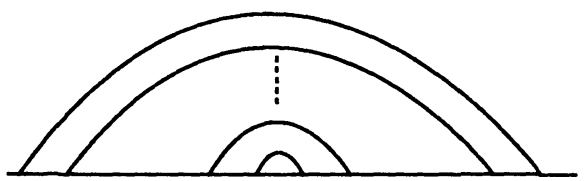

FIGURE 5 
which represents the configuration in Fig. 5. In fact we have:

COROLlARY 1.3. Using the binomial coefficients,

$$
b(n, k)=\frac{k}{n}\left(\begin{array}{c}
2 n-k-1 \\
n-1
\end{array}\right) .
$$

Proof. By Proposition 1.2, $b(n+1, k)-b(n+1, k+1)=b(n, k-1)$. And this recursive formula together with initial conditions $b(2,1)=$ $b(n, n)=1$ for all $n$ generates all $b(n, k)$ 's. But a computation shows that

$$
\frac{k}{n+1}\left(\begin{array}{c}
2 n-k+1 \\
n
\end{array}\right)-\frac{k+1}{n+1}\left(\begin{array}{c}
2 n-k \\
n
\end{array}\right)=\frac{k-1}{n}\left(\begin{array}{c}
2 n-k \\
n-1
\end{array}\right) .
$$

Let $\mathfrak{B}(n, k)$ be the set of sequences with initial entry $k$ and the remaining terms forming a restricted sequence of length $n-1$. We will sometimes write $(k, \alpha)$ with $\alpha$ restricted for such a sequence. Note that $|\mathfrak{B}(n, k)|=C_{n-1}$.

Let $V$ be the free $\mathbf{Z}[\delta]$ module generated by $D_{n}$ where $\delta$ is a variable. We define a bilinear form on $V \times V$. If $\alpha, \beta$ are two configurations in $D_{n}$, we can form the union of their respective disks along the boundary to obtain a configuration of circles in the 2-sphere. We denote this configuration in $S^{2}$ by $\alpha \cup \beta$. Let $c$ be the number of circles in $\alpha \cup \beta$; then $\langle\alpha, \beta\rangle=\delta^{c}$. Then we linearly extend this pairing to all elements in the free module. Lickorish first considered this symmetric bilinear form to give a more geometric and combinatorial proof of the existence of the 3-manifold invariant developed by Witten and Reshetikhin-Turaev. See [2], [3], [4] and [5]. So we call it Lickorish's bilinear form. We can also consider this a pairing of restricted sequences or of sequences since they correspond to configurations.

LEMMA 1.4. For $\alpha, \beta \in D_{n},\langle\alpha, \beta\rangle=\delta^{n}$ if and only if $\alpha=\beta$.

Proof. If $\alpha=\beta$ then each component of $\alpha \cup \beta$ consists of one arc of $\alpha$ and one of $\beta$ so $\alpha \cup \beta$ has $n$ components. If $\langle\alpha, \beta\rangle=\delta^{n}$ then each arc of $\alpha$ is in a separate component of $\alpha \cup \beta$. But if $\alpha \neq \beta$ then some arc of $\beta$ joins endpoints of two distinct arcs of $\alpha$ and these arcs are in the same component of $\alpha \cup \beta$.

TheOREM 1.5 (Properties of Lickorish's bilinear form). (1) Let $S$ be any subset of $D_{n}$. Then $\langle$,$\rangle is nondegenerate over the free \mathbf{Z}[\delta]$ module generated by $S$. 
$\tau_{2}$

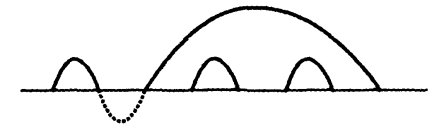

$\tau_{3}$
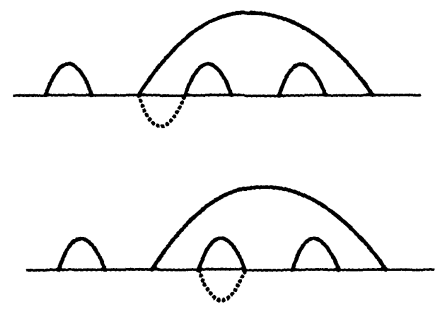

$=$

$=$

$=$
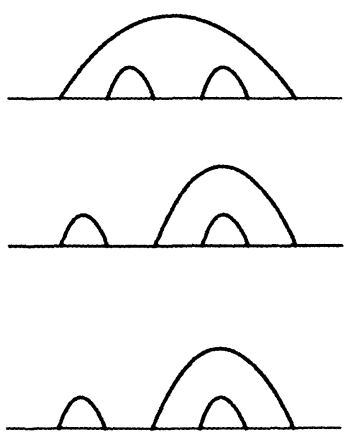

FIGURE 6

(2) Suppose $\alpha$ is any configuration in $D_{n}$. Then for any $b \in$ $\{1,2, \ldots, n\}$ with $\alpha \notin \mathfrak{B}(n, b)$, there is $a \beta \in \mathfrak{B}(n, b)$ such that $\delta\langle\alpha, \gamma\rangle=\langle\beta, \gamma\rangle$ for all $\gamma \in \mathfrak{B}(n, b)$.

(3) $\delta\langle\alpha, \varepsilon\rangle=\langle(a, \alpha),(a, \varepsilon)\rangle=\delta\langle(a \pm 1, \alpha),(a, \varepsilon)\rangle$ for all sequences $\varepsilon, \alpha$ whenever $a \pm 1$ makes sense.

(4) Suppose $(a, \alpha),(b, \beta)$ are restricted sequences of length $n$ and there is an $\eta \in \mathbf{Z}[\delta]$ such that $\langle(a, \alpha), \gamma\rangle=\eta\langle(b, \beta), \gamma\rangle$ for all $\gamma \in$ $\mathfrak{B}(n, a)$ with $\gamma \leq(a, \alpha)$.

(i) If $b=a, a \pm 1$ then $\alpha \leq \beta$.

(ii) If $b \neq a, a \pm 1$ then $\alpha<\beta$.

Before we begin the proof, we first define a set of maps

$$
\tau_{a}: D_{n} \rightarrow D_{n-1} \quad \text { for } a=1,2, \ldots, n .
$$

These mappings eliminate the $a$ th and the $(a+1)$ st points in $D_{n}$ by an inverse of a "finger move" as in Fig. 6.

Note that $\tau_{a}((a, \alpha))=\alpha$ for any sequence $\alpha$.

Proof of Theorem 1.5. (1) Suppose $\sum_{\alpha \in S} q_{\alpha} \alpha$ is an arbitrary element in the free $\mathbf{Z}[\delta]$ module generated by $S$. From among the $q_{\alpha}$, pick a $\beta$ so that the degree of $q_{\beta}$ is maximal. Then by Lemma 1.4, the degree of $\langle\beta, \beta\rangle$ is strictly greater than the degree $\langle\alpha, \beta\rangle$ for all $\alpha \neq \beta$. Therefore $\left\langle\sum_{\alpha \in S} q_{\alpha} \alpha, \beta\right\rangle$ has a nonvanishing term of degree $\left(n+\operatorname{deg} q_{\beta}\right)$.

(2) If $\alpha \notin \mathfrak{B}(n, b)$, then

$$
\delta\langle\alpha, \gamma\rangle=\left\langle\left(b, \tau_{b}(\alpha)\right), \gamma\right\rangle \quad \text { for } \gamma \in \mathfrak{B}(n, b)
$$


e. g. $b=4$
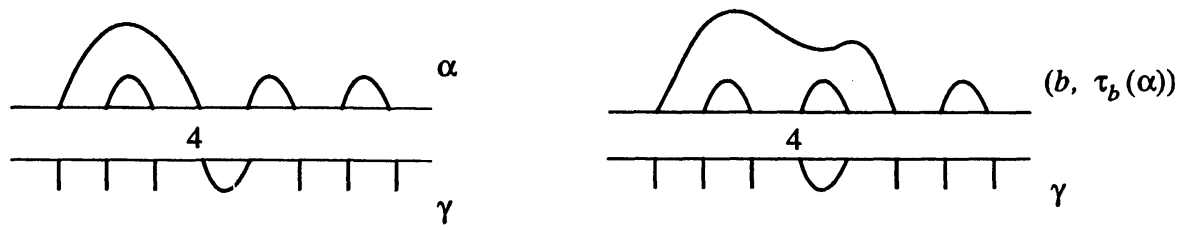

FIGURE 7
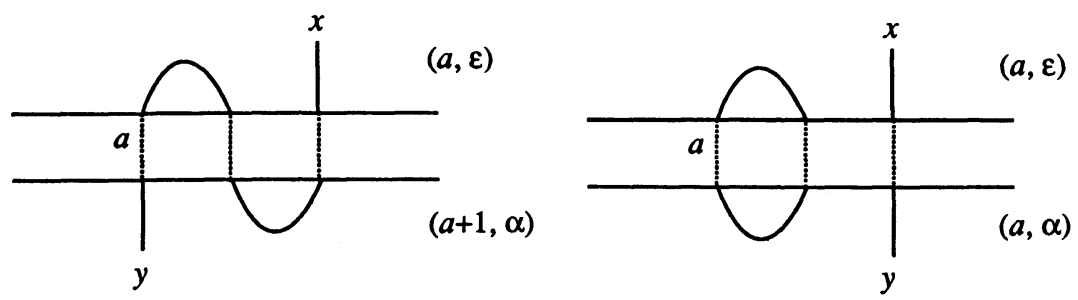

Figure 8

since the innermost arc at $b$ performs $\tau_{b}$ when joined to $\alpha$. See Fig. 7.

(3) $\tau_{a}(a, \alpha)=\tau_{a}(a+1, \alpha)=\tau_{a}(a-1, \alpha)=\alpha$. See Fig. 8 .

(4) It follows from Lemma 1.4 that

$$
\eta=\langle(a, \alpha),(a, \alpha)\rangle /\langle(b, \beta),(a, \alpha)\rangle=\delta^{k}
$$

for some $k \geq 0$. First suppose $b=a$ and so $(b, \beta) \in \mathfrak{B}(n, a)$. Let $S=\left\{\varepsilon \in D_{n-1} \mid \varepsilon \leq \alpha\right\}$. If $(b, \beta)<(a, \alpha)$, i.e., $\beta<\alpha$ then $\delta\left\langle\alpha-\delta^{k} \beta, \varepsilon\right\rangle=\left\langle(a, \alpha)-\delta^{k}(b, \beta),(a, \varepsilon)\right\rangle=0$ for all $\varepsilon \in S$. This contradicts property (1). Thus $(b, \beta) \geq(a, \alpha)$.

Suppose that $b=a \pm 1$. If $\beta<\alpha$ then this together with property (3) contradicts property (1). Thus $\beta \geq \alpha$.

Now suppose that $b \neq a, a \pm 1$. If $(b, \beta) \in \mathfrak{B}(n, a)$ then $b<a$ because $(b, \beta)$ is a restricted sequence. So $(b, \beta)<(a, \alpha)$, which again contradicts property $(1)$. Thus $(b, \beta) \notin \mathfrak{B}(n, a)$. We then have as in Fig. 9,

$$
\langle(b, \beta), \gamma\rangle=\left\langle\tau_{a}(b, \beta), \tau_{a} \gamma\right\rangle \quad \text { for all } \gamma \in \mathfrak{B}(n, a) \text { with } \gamma \leq(a, \alpha) \text {. }
$$

Then $\delta\left\langle\alpha, \tau_{a} \gamma\right\rangle=\langle(a, \alpha), \gamma\rangle=\delta^{k}\langle(b, \beta), \gamma\rangle=\delta^{k}\left\langle\tau_{a}(b, \beta), \tau_{a} \gamma\right\rangle$. Thus

$$
\delta\langle\alpha, \varepsilon\rangle=\delta^{k}\left\langle\tau_{a}(b, \beta), \varepsilon\right\rangle \text { for all } \varepsilon \in D_{n-1} \text { with } \varepsilon \leq \alpha .
$$

Thus we have $\tau_{a}(b, \beta) \geq \alpha$ by property (1). Let $\alpha_{1}, \beta_{1}$, and $\beta_{1}^{\prime}$ be the first entry of restricted sequences $\alpha, \beta$, and $\tau_{a}(b, \beta)$ respectively. 

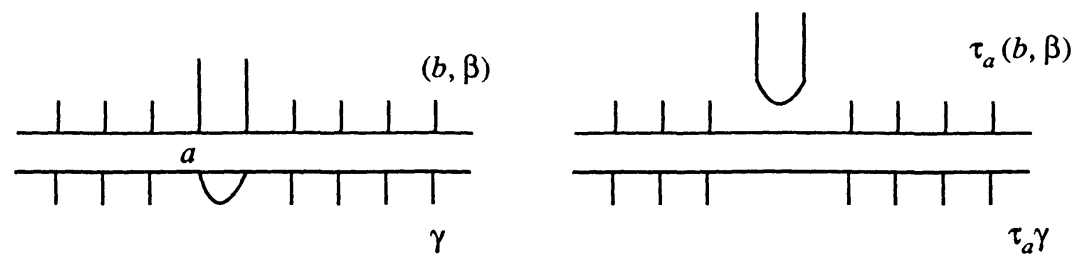

FIGURE 9

If $b<a-1$ then $\beta_{1}^{\prime} \leq b<a-1 \leq \alpha_{1}$ because $(a, \alpha)$ is a restricted sequence. So $\tau_{a}(b, \beta)<\alpha$ and this is a contradiction. Thus $b>$ $a+1$. Since $(b, \beta)$ has the first occurring innermost arc at $b, \beta_{1}^{\prime}=$ $b-2<\beta_{1}$ and so $\tau_{a}(b, \beta)<\beta$. Therefore $\alpha<\beta$.

2. Matrix manipulation. Let $T_{n}$ be the $(n \times n)$ tridiagonal matrix with $\delta$ in each diagonal element and 1 in each upper and lower superdiagonal. For example

$$
T_{5}=\left(\begin{array}{lllll}
\delta & 1 & 0 & 0 & 0 \\
1 & \delta & 1 & 0 & 0 \\
0 & 1 & \delta & 1 & 0 \\
0 & 0 & 1 & \delta & 1 \\
0 & 0 & 0 & 1 & \delta
\end{array}\right)
$$

Let $\Delta_{n}=\operatorname{det} T_{n}$, then it is a polynomial in $\delta$ for $n \geq 1$.

Proposition 2.1. (1) $\Delta_{n}=\delta \Delta_{n-1}-\Delta_{n-2}$ for $n \geq 3$.

(2) $\Delta_{n}=\prod_{k=1}^{n}\left(\delta-2 \cos \frac{k \pi}{n+1}\right)$.

Proof. (1) Compute $\Delta_{n}$ by expanding along the first row.

(2) Note that $\Delta_{n}$ is of degree $n$ and the coefficient of $\delta^{n}$ is 1 so that we must find the roots of $\Delta_{n}$. Since $\Delta_{n}=n+1$ when $\delta=2$ and $\Delta_{n}=(-1)^{n}(n+1)$ when $\delta=-2, \delta= \pm 2$ are not roots. We solve the recursion formula by a standard method. Let

$$
\alpha=\frac{\delta+\sqrt{\delta^{2}-4}}{2} \text { and } \beta=\frac{\delta-\sqrt{\delta^{2}-4}}{2}
$$

so that $\alpha \beta=1$ and $\alpha+\beta=\delta$. From the recursion we get $\Delta_{n}$ $\alpha \Delta_{n-1}=\beta\left(\Delta_{n-1}-\alpha \Delta_{n-2}\right)=\beta^{n}$. Similarly $\Delta_{n}-\beta \Delta_{n-1}=\alpha^{n}$. Then $(\alpha-\beta) \Delta_{n}=\alpha^{n+1}-\beta^{n+1}$. Thus $\Delta_{n}=0$ exactly when $\alpha \neq \beta$ and $\alpha^{n+1}=\beta^{n+1}$. Since $\beta<1<\alpha$ when $\delta>2$ and $\beta<-1<\alpha$ when $\delta<-2, \delta$ cannot be a root for $|\delta|>2$. Thus we may assume $|\delta|<2$ so $\delta=2 \operatorname{Re} \alpha=2 \operatorname{Re} \beta$. Also $\alpha^{n+1}=\beta^{n+1}$ is equivalent to $\alpha^{2 n+2}=1$. If we take $\alpha$ to be one among the first $n$ of $(2 n+2)$ th roots of unity, then $\alpha$ is not equal to $\beta$ which is now the conjugate 
of $\alpha$. Thus $\delta=2 \cos (k \pi /(n+1))$ for $k=1, \ldots, n$. Since they are all distinct, we found all of the roots of $\Delta_{n}=0$.

LEMMA 2.2. Let $A$ be a symmetric matrix over a ring and $A^{\prime}$ be obtained by deleting the last row and column. If $\operatorname{det} A^{\prime} \neq 0$, then $a$ series of row operations and the corresponding column operations within the ring convert $A$ into $\left(\begin{array}{cc}A^{\prime} & 0 \\ 0 & \operatorname{det} A^{\prime} \operatorname{det} A\end{array}\right)$.

Proof. Let

$$
\left(\begin{array}{c}
v_{1} \\
\vdots \\
v_{n}
\end{array}\right)
$$

be the last column of $A$. Let $y$ be the solution of the system of equations:

$$
A^{\prime} x=\operatorname{det} A^{\prime}\left(\begin{array}{c}
v_{1} \\
\vdots \\
v_{n-1}
\end{array}\right)
$$

Define

$$
E=\left(\begin{array}{cc}
I & -y \\
0 & \operatorname{det} A^{\prime}
\end{array}\right)
$$

Then

$$
E^{\operatorname{tr}} A E=\left(\begin{array}{cc}
A^{\prime} & 0 \\
0 & \operatorname{det} A^{\prime} \operatorname{det} A
\end{array}\right)
$$

REMARK. Applying row operations, one gets

$$
E^{\operatorname{tr}} A=\left(\begin{array}{cc} 
& v_{1} \\
A^{\prime} & \vdots \\
& v_{n-1} \\
0 & \operatorname{det} A
\end{array}\right) .
$$

Let $A(n)$ be the matrix representation of Lickorish's bilinear form $\langle$,$\rangle over the basis D_{n}$ ordered by restricted sequences. $A(n)$ consists of $n^{2}$ blocks of matrices $M_{i j}$ such that $M_{i j}$ represents $\langle$,$\rangle on$ $B(n, i) \times B(n, j)$. So $M_{i j}$ is a $b(n, i) \times b(n, j)$ matrix. Let $A(n, k)$ be the submatrix $\left(M_{i j}\right)_{i, j=1, \ldots, k}$ of $A(n)$. Thus $A(n, n)=A(n)$ and $A(n, 1)=\delta A(n-1)$ in this notation. By Theorem 1.5(3),

$$
A(n, 2)=\left(\begin{array}{cc}
\delta A(n-1) & A(n-1) \\
A(n-1) & \delta A(n-1)
\end{array}\right) .
$$

Thus we have the following proposition. 
Proposition 2.3. (1) $\operatorname{det} A(n, 1)=\Delta_{1}^{C_{n-1}} \operatorname{det} A(n-1)$.

(2) $\operatorname{det} A(n, 2)=\Delta_{2}^{C_{n-1}}(\operatorname{det} A(n-1))^{2}$.

Proof. Just calculate.

LEMMA 2.4. $A(n)$ and all of its principal minors have nonzero determinants.

Proof. By Lemma 1.4, only the diagonal entries of $A(n)$ have the highest degree $n$. Thus the term $\delta^{n C_{n}}$ in the determinant of $A(n)$ has the coefficient 1 . And the same argument applies to all principal minors.

Given a matrix $M, M^{\langle p\rangle}$ denotes the matrix obtained from $M$ by deleting the last $p$ rows and columns. And $A(n, k)^{\langle\bar{p}\rangle}$ denotes the matrix $\left(M_{i j}^{\langle p\rangle}\right)_{i, j=1, \ldots, k}$.

LEMMA 2.5. (1) For $0 \leq p \leq b(n, k)-1$, we have the following recursion formula:

$$
\operatorname{det} A(n, k)^{\langle\bar{p}\rangle}=\Delta_{k}\left(\begin{array}{c}
\operatorname{det} A(n-1)^{\langle p\rangle} \\
\operatorname{det} A(n-1)^{\langle p+1\rangle}
\end{array}\right)^{k} \operatorname{det} A(n, k)^{\langle\overline{p+1}\rangle} .
$$

(2) For $2 \leq j \leq k-1$ and $b(n, j+1) \leq p \leq b(n, j)-1$, we have the following recursion formula:

$$
\operatorname{det} A(n, k)^{\langle\bar{p}\rangle}=\Delta_{j}\left(\frac{\operatorname{det} A(n-1)^{\langle p\rangle}}{\operatorname{det} A(n-1)^{\langle p+1\rangle}}\right)^{j} \operatorname{det} A(n, k)^{\langle\overline{p+1}\rangle} .
$$

In order to help the understanding of the proof given below, we will describe some of the properties of $A(n)$ that reflect the properties of Lickorish's bilinear form in Theorem 1.5. Let $\mathfrak{M}_{i j}$ be the matrix representing $\langle$,$\rangle on \mathfrak{B}(n, i) \times \mathfrak{B}(n, j)$. Then property (2) in Theorem 1.5 means that each column of $\mathfrak{M}_{i j}$ is equal to either one of columns of $\mathfrak{M}_{i i}$ or $\delta^{-1}$ times one of the columns. Property (3) implies that $\mathfrak{M}_{i i}=\delta A(n-1)$ and $\mathfrak{M}_{i(i \pm 1)}=A(n-1)$. Furthermore the last column of $\mathfrak{M}_{i i}$ is independent of every column in the blocks $\mathfrak{M}_{i j}$ for $j \neq i, i \pm 1$. This can be seen through property (4) since unrestricted sequences (i.e., repeated configurations) always appear first in the sets $\mathfrak{B}(n, k)$ for $k \geq 3$. Then row operations as in Lemma 2.2 with $A=A(n-1)$ convert the last row of $\mathfrak{M}_{i i}$ into $(0, \ldots, 0, \delta \operatorname{det} A(n-1)), M_{i(i \pm 1)}$ into $(0, \ldots, 0, \operatorname{det} A(n-1))$, and $\mathfrak{M}_{i j}$ for $j \neq i, i \pm 1$ into $(0, \ldots, 0,0)$. 
The matrix $\left(\mathfrak{M}_{i j}\right)_{i, j=1, \ldots, n}$ of the blocks has repeated rows due to the presence of unrestricted sequences. $A(n)$ then is obtained from this matrix by deleting repeated rows and corresponding columns. From $\mathfrak{M}_{i j}$ one would delete the first $\sum_{k=1}^{i-2} b(n-1, k)$ rows and $\sum_{k=1}^{j-2} b(n-1, k)$ columns so an undeleted column in $\mathfrak{M}_{i j}$ does not change its position when counted from the rear. Let $\mathfrak{B}(n, i)^{\langle p\rangle}$ and $B(n, i)^{\langle p\rangle}$ denote $\mathfrak{B}(n, i)$ and $B(n, i)$ with the last $p$ configurations deleted. Consider the matrix given by $\langle$,$\rangle on \mathfrak{B}(n, i)^{\langle p\rangle} \times$ $\bigcup_{j=1}^{n} B(n, j)^{\langle p\rangle}$. Any multiple of the column corresponding to the last element of $B(n, i)^{\langle p\rangle}$ appears only at the spots corresponding to the last elements of $B(n, i \pm 1)^{\langle p\rangle}$. By property (4) any other multiples were eliminated in the $p$ deletions since they occur nearer the rear of their respective $\mathfrak{B}(n, i)^{\langle p\rangle} \times B(n, j)^{\langle p\rangle}$ block.

In the matrix $A(n)$ there is still a minor which is $\mathfrak{M}_{i j}$; however it does not appear as a solid block since some of its configurations have innermost arcs which occur before the $i$ th spot. However one can perform the desired row operations by borrowing the missing rows from the blocks above. One may do similar operations on $A(n)^{\langle\bar{p}\rangle}$.

Proof of Lemma 2.5. (1) Let $E$ be the matrix as in the proof of Lemma 2.2 such that

$E^{\operatorname{tr}} A(n-1)^{\langle p\rangle} E=\left(\begin{array}{cc}A(n-1)^{\langle p+1\rangle} & 0 \\ 0 & \operatorname{det} A(n-1)^{\langle p+1\rangle} \operatorname{det} A(n-1)^{\langle p\rangle}\end{array}\right)$.

We may assume that the entries of $E$ are indexed by the first $C_{n-1}-p$ elements in $D_{n-1}$ that is ordered by the restricted sequences. Consider the set $\mathfrak{S}$ of sequences $(i, \alpha)$ for $i=1, \ldots, k$ and the first $C_{n-1}-p$ restricted sequences $\alpha$ in $D_{n-1}$. There is an obvious equivalence relation in which two sequences are equivalent if they represent the same configuration. Mod out $\mathfrak{S}$ by this relation and we obtain a subset $S$ of $D_{n}$. For $i=1, \ldots, k$ define a matrix $E_{i}$ whose entries are indexed by $S$. The $([(i, \alpha)],[(i, \beta)])$ th entry of $E_{i}$ is equal to the $(\alpha, \beta)$ th entry of $E$ for all elements $[(i, \alpha)],[(i, \beta)]$ of $S$. All other diagonal entries of $E_{i}$ are 1 and all other off-diagonal entries are 0 . Hence $E_{1}$ is the identity except in the upper $\left(C_{n-1}-p\right) \times\left(C_{n-1}-p\right)$ corner where it is $E$. And $E_{i}$ is obtained from $E_{1}$ by permuting rows and corresponding columns. Perform row operations $E_{1}^{\text {tr }}$ to $A(n, k)^{\langle\bar{p}\rangle}$ and denote the blocks of $E_{1}^{\operatorname{tr}} A(n, k)^{\langle\bar{p}\rangle}$ by $\left(G_{i j}\right)$. Then the last row of $G_{12}$ consists of zeros except the last entry because the $(1,2)$ th block of $A(n, k)^{\langle\bar{p}\rangle}$ is exactly equal to $A(n-1)^{\langle p\rangle}$. And by Theorem 1.5(3), $G_{11}=\delta G_{12}$. Theorem 1.5(2) and (4) say 
that every column of the $(1,3)$ th $, \ldots,(1, k)$ th blocks of $A(n, k)^{\langle\bar{p}\rangle}$ is equal to one of the columns of the $(1,2)$ th block of $A(n, k)^{\langle\bar{p}\rangle}$ which is not the last. Thus the last rows of $G_{13}, \ldots, G_{1 k}$ are all zero. We now perform additional row operations $E_{2}^{\text {tr }}, \ldots, E_{k}^{\text {tr }}$ and all the corresponding column operations. Then the resulting matrix $E_{k}^{\operatorname{tr}} \cdots E_{1}^{\operatorname{tr}} A(n, k)^{\langle\bar{p}\rangle} E_{1} \cdots E_{k}$ looks like

$$
\left(\begin{array}{lcllllll} 
& 0 & & 0 & & 0 & & 0 \\
M_{11}^{\langle p+1\rangle} & \vdots & M_{12}^{\langle p+1\rangle} & \vdots & M_{13}^{\langle p+1\rangle} & \vdots & & \vdots \\
& 0 & & 0 & & 0 & & 0 \\
0 \cdots 0 & \delta \xi & 0 \cdots 0 & \xi & 0 \cdots 0 & 0 & 0 \cdots & 0 \\
& 0 & & 0 & & 0 & & 0 \\
M_{21}^{\langle p+1\rangle} & \vdots & M_{22}^{\langle p+1\rangle} & \vdots & M_{23}^{\langle p+1\rangle} & \vdots & & \vdots \\
& 0 & & 0 & & 0 & & 0 \\
0 \cdots 0 & \xi & 0 \cdots 0 & \delta \xi & 0 \cdots 0 & \xi & 0 \cdots & 0 \\
& 0 & & 0 & & 0 & & 0 \\
M_{31}^{\langle p+1\rangle} & \vdots & M_{32}^{\langle p+1\rangle} & \vdots & M_{33}^{\langle p+1\rangle} & \vdots & & \vdots \\
& 0 & & 0 & & 0 & & 0 \\
0 \cdots 0 & 0 & 0 \cdots 0 & \xi & 0 \cdots 0 & \delta \xi & \cdots & 0 \\
& 0 & & 0 & & & & 0 \\
& \vdots & & \vdots & & \vdots & & \vdots \\
0 \cdots 0 & 0 & 0 \cdots 0 & 0 & 0 \cdots 0 & 0 & 0 \cdots & \delta \xi
\end{array}\right)
$$

and $\xi=\operatorname{det} A(n-1)^{\langle p+1\rangle} \operatorname{det} A(n-1)^{\langle p\rangle}$. By permuting rows and corresponding columns, the matrix becomes

$$
\left(\begin{array}{cc}
\xi T_{k} & 0 \\
0 & A(n, k)^{\langle\overline{p+1}\rangle}
\end{array}\right) .
$$

But

$$
\operatorname{det} E_{i}=\operatorname{det} A(n-1)^{\langle p+1\rangle}
$$

and

$$
\operatorname{det}\left(\xi T_{k}\right)=\Delta_{k}\left(\operatorname{det} A(n-1)^{\langle p+1\rangle} \operatorname{det} A(n-1)^{\langle p\rangle}\right)^{k} .
$$

(2) The proof is similar. The only difference is that $A(n, k)^{\langle\bar{p}\rangle}$ now has $j^{2}$ blocks so we try to factor the tridiagonal matrix $T_{j}$ out from it.

LEMMA 2.6. For $3 \leq k \leq n$, we have the following recursion formulae:

$$
\operatorname{det} A(n-1)=\Delta_{k}^{b(n, k)}\left(\frac{\operatorname{det} A(n-1)}{\operatorname{det} A(n-1, k-2)}\right)^{k} \operatorname{det} A(n-1)^{\langle\overline{b(n, k)}\rangle},
$$


and when $2 \leq j \leq k-1$,

$$
\begin{aligned}
\operatorname{det} A & (n-1)^{\langle\overline{b(n, j+1)}\rangle} \\
& =\Delta_{j}^{b(n, j)-b(n, j+1)}\left(\frac{\operatorname{det} A(n-1, j-1)}{\operatorname{det} A(n-1, j-2)}\right)^{j} \operatorname{det} A(n-1)^{\langle\overline{b(n, j)\rangle}} .
\end{aligned}
$$

Proof. We successively apply the recursion formula (1) in Lemma 2.5. Then

$$
\begin{aligned}
\operatorname{det} A(n, k)= & \Delta_{k}\left(\frac{\operatorname{det} A(n-1)}{\operatorname{det} A(n-1)^{\langle 1\rangle}}\right)^{k} \operatorname{det} A(n-1)^{\langle\overline{1}\rangle} \\
= & \Delta_{k}^{2}\left(\frac{\operatorname{det} A(n-1)}{\operatorname{det} A(n-1)^{\langle 1\rangle}}\right)^{k}\left(\frac{\operatorname{det} A(n-1)^{\langle 1\rangle}}{\operatorname{det} A(n-1)^{\langle 2\rangle}}\right)^{k} \operatorname{det} A(n, k)^{\langle\overline{2}\rangle} \\
& \ldots \\
= & \Delta_{k}^{b(n, k)}\left(\frac{\operatorname{det} A(n-1)}{\operatorname{det} A(n-1)^{\langle 1\rangle}}\right)^{k}\left(\frac{\operatorname{det} A(n-1)^{\langle 1\rangle}}{\operatorname{det} A(n-1)^{\langle 2\rangle}}\right)^{k} \\
& \ldots\left(\frac{\operatorname{det} A(n-1)^{\langle b(n, k)-1\rangle}}{\operatorname{det} A(n-1)^{\langle b(n, k)\rangle}}\right)^{k} \operatorname{det} A(n, k)^{\langle\overline{b(n, k)\rangle}} \\
= & \Delta_{k}^{b(n, k)}\left(\frac{\operatorname{det} A(n-1)}{\operatorname{det} A(n-1)^{\langle b(n, k)\rangle}}\right)^{k} \operatorname{det} A(n, k)^{\langle\overline{b(n, k)\rangle}} .
\end{aligned}
$$

But $A(n-1)^{\langle b(n, k)\rangle}=A(n-1, k-2)$ because

$$
b(n, k)=\sum_{i=k-1}^{n-1} b(n-1, i)
$$

The other formula can be shown by using the formula (2) in Lemma 2.5.

THEOREM 2.7. For $3 \leq k \leq n$, we have the following recursion formula:

$\operatorname{det} A(n, k)$

$$
=\frac{\Delta_{k}^{b(n, k)} \Delta_{k-1}^{b(n, k-1)-b(n, k)} \cdots \Delta_{2}^{b(n, 2)-b(n, 3)}(\operatorname{det} A(n-1))^{k}}{(\operatorname{det} A(n-1, k-2))(\operatorname{det} A(n-1, k-3)) \cdots(\operatorname{det} A(n-1,1))} .
$$


Proof. We recursively use the formulae in Lemma 2.6. $\operatorname{det} A(n, k)$

$$
\begin{aligned}
= & \Delta_{k}^{b(n, k)}\left(\frac{\operatorname{det} A(n-1)}{\operatorname{det} A(n-1, k-2)}\right)^{k} \operatorname{det} A(n, k)^{\langle\overline{b(n, k)\rangle}} \\
= & \Delta_{k}^{b(n, k)} \Delta_{k-1}^{b(n, k-1)-b(n, k)}\left(\frac{\operatorname{det} A(n-1)}{\operatorname{det} A(n-1, k-2)}\right)^{k} \\
& \cdot\left(\frac{\operatorname{det} A(n-1, k-2)}{\operatorname{det} A(n-1, k-3)}\right)^{k-1} \operatorname{det} A(n, k)^{\langle\overline{\langle(n, k-1)\rangle}} \\
& \ldots \\
= & \Delta_{k}^{b(n, k)} \Delta_{k-1}^{b(n, k-1)-b(n, k)} \cdots \Delta_{3}^{b(n, 3)-b(n, 4)}\left(\frac{\operatorname{det} A(n-1)}{\operatorname{det} A(n-1, k-2)}\right)^{k} \\
& \cdot\left(\frac{\operatorname{det} A(n-1, k-2)}{\operatorname{det} A(n-1, k-3)}\right)^{k-1} \\
& \ldots\left(\frac{\operatorname{det} A(n-1,2)}{\operatorname{det} A(n-1,1)}\right)^{3} \operatorname{det} A(n, k)^{\langle\overline{b(n, 3)}\rangle} \\
= & \frac{\Delta_{k}^{b(n, k)} \Delta_{k-1}^{b(n, k-1)-b(n, k)} \ldots \Delta_{3}^{b(n, 3)-b(n, 4)}(\operatorname{det} A(n-1))^{k}}{(\operatorname{det} A(n-1, k-2))(\operatorname{det} A(n-1, k-3)) \cdots(\operatorname{det} A(n-1,2))} \\
& \cdot \frac{\operatorname{det} A(n, k)^{\langle\overline{b(n, 3)\rangle}}}{(\operatorname{det} A(n-1,1))^{3}} \cdot
\end{aligned}
$$

But

$$
\begin{aligned}
A(n, k)^{\langle\overline{b(n, 3)\rangle}} & =A(n, 2)^{\langle\overline{b(n, 3)\rangle}} \\
& =\left(\begin{array}{cc}
\delta A(n-1)^{\langle b(n, 3)\rangle} & A(n-1)^{\langle b(n, 3)\rangle} \\
A(n-1)^{\langle b(n, 3)\rangle} & \delta A(n-1)^{\langle b(n, 3)\rangle}
\end{array}\right) .
\end{aligned}
$$

Since $A(n-1)^{\langle b(n, 3)\rangle}=A(n-1,1)$,

$$
\begin{aligned}
\operatorname{det} A(n, k)^{\langle\overline{b(n, 3)\rangle}} & =\Delta_{2}^{b(n-1,1)}(\operatorname{det} A(n-1,1))^{2} \\
& =\Delta_{2}^{b(n, 2)-b(n, 3)}(\operatorname{det} A(n-1,1))^{2}
\end{aligned}
$$

REMARK. By inserting the factor $\Delta_{1}^{b(n, 1)-b(n, 2)}$, which is 1 , into the formula in Theorem 2.7, we obtain a recursion formula that works for all $k=1, \ldots, n$. See Proposition 2.3.

COROLlaRY 2.8. The $\operatorname{det} A(n)$ vanishes at twice the real part of any primitive $2(n+1)$ st root of unity and $\operatorname{det} A(m, k)$ for $1 \leq m \leq n-1$ and $1 \leq k \leq n-1$ never vanishes at these values. 
Proof. The recursion formula of Theorem 2.7 shows that the determinants $\operatorname{det} A(m, k)$ for $1 \leq m \leq n$ and $1 \leq k \leq m$ can be written as a product of positive or negative powers of $\Delta_{1}, \ldots, \Delta_{n}$. It also shows that $\operatorname{det} A(n)$ contains the factor $\Delta_{n}$ exactly once and all the other determinants of lower indexes do not contain the factor $\Delta_{n}$. Therefore $2 \cos \frac{k \pi}{n+1}$ must be a root of $\operatorname{det} A(n)$ if $k$ is relatively prime to $n+1$.

COROllary 2.9. After setting $\delta$ to be twice the real part of any primitive $2(n+1)$ st root of unity, Lickorish's pairing can be considered as a symmetric bilinear form over the real (or complex) vector space with a basis $D_{n}$. Then the basis element $\alpha=(n, n-1, \ldots, 2,1)$ has the property that there is a linear combination $\sum_{\beta \neq \alpha} q_{\beta} \beta$ of basis elements other than $\alpha$ such that $\langle\alpha, \gamma\rangle=\left\langle\sum_{\beta \neq \alpha} q_{\beta} \beta, \gamma\right\rangle$ for all $\gamma$ in the vector space.

Proof. The last row of $A(n)$ corresponds to $\alpha$ and $A(n)^{\langle 1\rangle}=$ $A(n, n-1)$. By Corollary 2.8, $A(n)$ is singular but $A(n, n-1)$ is nonsingular. Thus it follows from Lemma 2.2. In fact, $q_{\beta}$ 's are equal up to sign to the $(\alpha, \beta)$ th cofactor of $A(n)$ divided by $\operatorname{det} A(n, n-1)$.

REMARK. In fact the last elements of each block $B(n, k)$ of $D_{n}$ as well as the rotations of the configuration $(n, n-1, \ldots, 1)$ have the property of Corollary 2.9.

COROLLARY 2.10. We have the following recursive formula:

$\operatorname{det} A(n)=\prod_{i=1}^{[(n+1) / 2]}(\operatorname{det} A(n-i))^{(-1)^{i-1}\left(\begin{array}{c}n-i+1 \\ i\end{array}\right)} \prod_{i=0}^{[(n-1) / 2]}\left(\frac{\Delta_{n-i}}{\Delta_{i}}\right)^{b(n-i, n-2 i)}$ where $\Delta_{0}=0$.

Proof. One can derive this from the formula in Theorem 2.7 using the following identities:

$$
\left(\begin{array}{c}
n-i+1 \\
i
\end{array}\right)=\sum_{k_{1}=1}^{n-2 i+2} \sum_{k_{2}=1}^{k_{1}} \cdots \sum_{k_{i}=1}^{k_{i-1}} 1
$$

and

$$
b(n, k)-b(n, k+1)=b(n-1, k-1) .
$$


Let $d(n, j)$ denote the exponent of $\Delta_{j}$ in $\operatorname{det} A(n)$. It is not hard to see that $d(n, j)$ is well defined for $j \geq 1$.

Corollary 2.11. For $j \geq 1$, we have that

$$
\sum_{i=0}^{[(n+1) / 2]}(-1)^{i}\left(\begin{array}{c}
n-i+1 \\
i
\end{array}\right) d(n-i, j)=b(j, 2 j-n)
$$

where $b(n, k)=-b(n-k,-k)$ for $k<0$ and $b(n, 0)=b(n, k)=0$ for $k>n$.

Proof. Immediate from Corollary 2.10.

REMARK. It is interesting to note that the Catalan numbers satisfy the similar formula:

$$
\begin{aligned}
& \sum_{i=0}^{[(n+1) / 2]}(-1)^{i}\left(\begin{array}{c}
n-i+1 \\
i
\end{array}\right) C_{j-i+1} \\
& \quad=b(j+2, n+2) \text { for } j \geq\left[\frac{n+1}{2}\right] .
\end{aligned}
$$

This formula can be proved by recalling that $b(n, k)$ is the number of configurations in $D_{n}$ that the first innermost arc occurs at the $k$ th point and by applying the inclusion-exclusion principle.

The following theorem shows that $\operatorname{det} A(n)$ is generated by a simple rule.

THEOREM 2.12. For $j \geq 1$, we have that

$$
d(n, j)=d(n-1, j-1)+2 d(n-1, j)+d(n-1, j+1)
$$

where $d(n, 0)=2 C_{n}-C_{n+1}=-\frac{4}{n+2}\left(\begin{array}{c}2 n-1 \\ n+1\end{array}\right)$.

Proof. By the remark following Corollary 2.11, the formula in Corollary 2.11 holds for $j=0$ if we set $b(0, k)=-b(-k,-k)=-1$ for $k<0$. Use an induction on $(n, j)$ with lexicographic order. Since $d(1,0)=2 C_{1}-C_{2}=0, d(2,1)=d(1,0)+2 d(1,1)+d(1,2)$. 
From the formula in Corollary 2.11 ,

$$
d(n, j)=b(j, 2 j-n)+\sum_{i=1}^{[(n+1) / 2]}(-1)^{i-1}\left(\begin{array}{c}
n-i+1 \\
i
\end{array}\right) d(n-i, j) .
$$

By the induction hypothesis and the identity $\left(\begin{array}{c}n-i+1 \\ i\end{array}\right)=\left(\begin{array}{c}n-i \\ i\end{array}\right)+\left(\begin{array}{c}n-i \\ i-1\end{array}\right)$,

$$
\begin{aligned}
& \sum_{i=1}^{[(n+1) / 2]}(-1)^{i-1}\left(\begin{array}{c}
n-i+1 \\
i
\end{array}\right) d(n-i, j) \\
& =\sum_{i=1}^{[(n+1) / 2]}(-1)^{i-1}\left(\begin{array}{c}
n-i \\
i
\end{array}\right)(d(n-i-1, j-1)+2 d(n-i-1, j) \\
& +d(n-i-1, j+1)) \\
& +\sum_{i=1}^{[(n+1) / 2]}(-1)^{i-1}\left(\begin{array}{c}
n-i \\
i-1
\end{array}\right)(d(n-i-1, j-1)+2 d(n-i-1, j) \\
& +d(n-i-1, j+1)) \\
& =\sum_{i=1}^{[n / 2]}(-1)^{i-1}\left(\begin{array}{c}
n-i \\
i
\end{array}\right)(d(n-i-1, j-1)+2 d(n-i-1, j) \\
& +d(n-i-1, j+1)) \\
& +\sum_{i=0}^{[(n-1) / 2]}(-1)^{i}\left(\begin{array}{c}
n-i-1 \\
i
\end{array}\right) \\
& \times(d(n-i-2, j-1)+2 d(n-i-2, j) \\
& +d(n-i-2, j+1)) \\
& =d(n-1, j-1)-b(j-1,2 j-n-1) \\
& +2 d(n-1, j)-2 b(j, 2 j-n+1)+d(n-1, j+1) \\
& -b(j+1,2 j-n+3)+b(j-1,2 j-n) \\
& +2 b(j, 2 j-n+2)+b(j+1,2 j-n+4) \\
& =-b(j, 2 j-n)+d(n-1, j-1)+2 d(n-1, j) \\
& +d(n-1, j+1) \text {. }
\end{aligned}
$$

The last equality is achieved by several uses of the identity

$$
b(n, k)-b(n, k+1)=b(n-1, k-1)
$$

for all integer $k$ and all $n \geq 2$.

One can now easily generate $\operatorname{det} A(n)$ by using the rule in Theorem 2.12 as in the following table. The term $\Delta_{0}=1$ is inserted for a 
computational purpose.

$$
\begin{aligned}
& \operatorname{det} A(1)=\Delta_{0}^{0} \quad \Delta_{1} \\
& \operatorname{det} A(2)=\Delta_{0}^{-1} \quad \Delta_{1}^{2} \quad \Delta_{2} \\
& \operatorname{det} A(3)=\Delta_{0}^{-4} \quad \Delta_{1}^{4} \quad \Delta_{2}^{4} \quad \Delta_{3} \\
& \operatorname{det} A(4)=\Delta_{0}^{-14} \quad \Delta_{1}^{8} \quad \Delta_{2}^{13} \quad \Delta_{3}^{6} \quad \Delta_{4} \\
& \operatorname{det} A(5)=\Delta_{0}^{-48} \quad \Delta_{1}^{15} \quad \Delta_{2}^{40} \quad \Delta_{3}^{26} \quad \Delta_{4}^{8} \quad \Delta_{5} \\
& \operatorname{det} A(6)=\Delta_{0}^{-165} \Delta_{1}^{22} \quad \Delta_{2}^{121} \Delta_{3}^{100} \Delta_{4}^{43} \Delta_{5}^{10} \Delta_{6} \\
& \operatorname{det} A(7)=\Delta_{0}^{-572} \Delta_{1}^{0} \quad \Delta_{2}^{364} \Delta_{3}^{364} \Delta_{4}^{196} \Delta_{5}^{64} \Delta_{6}^{12} \Delta_{7} \\
& \operatorname{det} A(8)=\Delta_{0}^{-2002} \Delta_{1}^{-208} \Delta_{2}^{1092} \Delta_{3}^{1288} \Delta_{4}^{820} \Delta_{5}^{336} \Delta_{6}^{89} \Delta_{7}^{14} \Delta_{8}
\end{aligned}
$$

REMARK. Notice that the exponents of $\Delta_{i}$ may be negative; however $\operatorname{det} A(n)$ is a polynomial in $\delta$. The negative exponents arise since the $\Delta_{i}$ 's are not relatively prime to each other. In fact the factor $\delta-2 \cos \frac{k \pi}{i+1}$ of $\Delta_{i}$ is also a factor of $\Delta_{j}$ if $i+1$ divides $j+1$. Moreover, if $k$ is relatively prime to $i+1$, then the converse holds. For example $\delta$ is a factor of $\Delta_{2 i+1}$ for all $i$ and $\delta^{2}-1$ is a factor of $\Delta_{3 i+2}$ for all $i$. R. A. Litherland has shown that the exponent of $\delta$ in $\operatorname{det} A(n)$ is $C_{n}$ and that the exponent of $\delta^{2}-1$ is $C_{n}-1$.

\section{REFERENCES}

[1] R. A. Brualdi, Introductory Combinatorics, North-Holland, New York, 1977.

[2] R. C. Kirby and P. Melvin, Evaluations of the 3-manifold invariants of Witten and Reshetikhin-Turaev for $\mathrm{sl}(2, \mathrm{C})$, (to appear).

[3] W. B. R. Lickorish, 3-manifold invariants from the combinatorics of the Jones polynomial, preprint.

[4] N. Yu. Reshetikhin and V. G. Turaev, Invariants of 3-manifolds via link polynomials and quantum groups, Invent. Math., (to appear).

[5] E. Witten, Quantum field theory and Jones' polynomial, Comm. Math. Phys., 121 (1989), 351-399.

Received February 23, 1990 and in revised form April 5, 1990. The first author was partially supported by a Korea Science and Engineering Foundation Postdoctoral Fellowship, and the second author partially supported by a Louisiana Education Quality Support Fund grant LEQSF(87-89)-RD-A-8.

Korea Institute of TeChNOLOgy

TAEJON, 305-701, KOREA

Currently visiting: Louisiana State University

Baton Rouge, LA 70803

AND

Louisiana State UNIVERSITY

BATON Rouge, LA 70803 


\section{PACIFIC JOURNAL OF MATHEMATICS EDITORS}

\author{
V. S. VARAdaraJan \\ (Managing Editor) \\ University of California \\ Los Angeles, CA 90024-1555-05 \\ Herbert Clemens \\ University of Utah \\ Salt Lake City, UT 84112 \\ THOMAS ENRIGHT \\ University of California, San Diego \\ La Jolla, CA 92093
}

R. FINN

Stanford University

Stanford, CA 94305

Hermann FlaschKa

University of Arizona

Tucson, AZ 85721

VAUGHAN F. R. Jones

University of California

Berkeley, CA 94720

STEVEN KerckHofF

Stanford University

Stanford, CA 94305

\section{C. MOORE}

University of California

Berkeley, CA 94720

Martin ScharlemanN

University of California

Santa Barbara, CA 93106

HAROLd STARK

University of California, San Diego

La Jolla, CA 92093
R. ARENS
E. F. BECKENBACH
F. WoLF
K. YoshidA
ASSOCIATE EDITORS

\begin{tabular}{ll}
\multicolumn{1}{c}{$(1906-1982)$} & \multicolumn{1}{c}{$(1904-1989)$} \\
SUPPORTING & INSTITUTIONS \\
UNIVERSITY OF ARIZONA & UNIVERSITY OF OREGON \\
UNIVERSITY OF BRITISH COLUMBIA & UNIVERSITY OF SOUTHERN CALIFORNIA \\
CALIFORNIA INSTITUTE OF TECHNOLOGY & STANFORD UNIVERSITY \\
UNIVERSITY OF CALIFORNIA & UNIVERSITY OF HAWAII \\
MONTANA STATE UNIVERSITY & UNIVERSITY OF TOKYO \\
UNIVERSITY OF NEVADA, RENO & UNIVERSITY OF UTAH \\
NEW MEXICO STATE UNIVERSITY & WASHINGTON STATE UNIVERSITY \\
OREGON STATE UNIVERSITY & UNIVERSITY OF WASHINGTON
\end{tabular}

The Supporting Institutions listed above contribute to the cost of publication of this Journal, but they are not owners or publishers and have no responsibility for its content or policies.

Mathematical papers intended for publication in the Pacific Journal of Mathematics should be in typed form or offset-reproduced (not dittoed), double spaced with large margins. Please do not use built up fractions in the text of the manuscript. However, you may use them in the displayed equations. Underline Greek letters in red, German in green, and script in blue. The first paragraph must be capable of being used separately as a synopsis of the entire paper. In particular it should contain no bibliographic references. Please propose a heading for the odd numbered pages of less than 35 characters. Manuscripts, in triplicate, may be sent to any one of the editors. Please classify according to the 1991 Mathematics Subject Classification scheme which can be found in the December index volumes of Mathematical Reviews. Supply name and address of author to whom proofs should be sent. All other communications should be addressed to the managing editor, or Elaine Barth, University of California, Los Angeles, California 90024-1555-05.

There are page-charges associated with articles appearing in the Pacific Journal of Mathematics. These charges are expected to be paid by the author's University, Government Agency or Company. If the author or authors do not have access to such Institutional support these charges are waived. Single authors will receive 50 free reprints; joint authors will receive a total of 100 free reprints. Additional copies may be obtained at cost in multiples of 50 .

The Pacific Journal of Mathematics (ISSN 0030-8730) is published monthly except for July and August. Regular subscription rate: $\$ 190.00$ a year (10 issues). Special rate: $\$ 95.00$ a year to individual members of supporting institutions.

Subscriptions, orders for numbers issued in the last three calendar years, and changes of address should be sent to Pacific Journal of Mathematics, P.O. Box 969, Carmel Valley, CA 93924, U.S.A. Old back numbers obtainable from Kraus Periodicals Co., Route 100, Millwood, NY 10546.

The Pacific Journal of Mathematics at P.O. Box 969, Carmel Valley, CA 93924 (ISSN 0030-8730) is published monthly except for July and August. Second-class postage paid at Carmel Valley, California 93924, and additional mailing offices. Postmaster: send address changes to Pacific Journal of Mathematics, P.O. Box 969, Carmel Valley, CA 93924.

PUBLISHED BY PACIFIC JOURNAL OF MATHEMATICS, A NON-PROFIT CORPORATION Copyright (C) 1991 by Pacific Journal of Mathematics 


\section{Pacific Journal of Mathematics}

Vol. 149, No. $2 \quad$ June, 1991

Manuel Alfaro Garcia, Mark Conger and Kenneth Hodges, The structure

of singularities in $\Phi$-minimizing networks in $\mathbf{R}^{2}$

Werner Balser, Dependence of differential equations upon parameters in

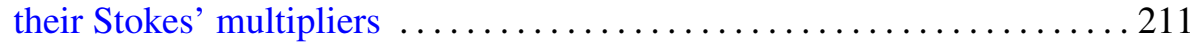

Enrico Casadio Tarabusi and Stefano Trapani, Envelopes of holomorphy

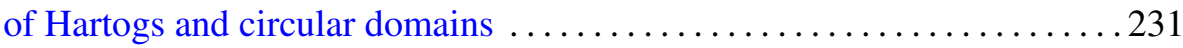

Hermann Flaschka and Luc Haine, Torus orbits in $G / P \ldots \ldots \ldots \ldots 251$

Gyo Taek Jin, The Cochran sequences of semi-boundary links .......... 293

Yasuyuki Kawahigashi, Cohomology of actions of discrete groups on

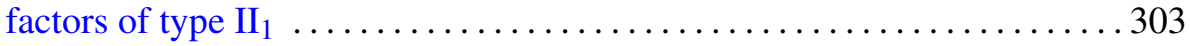

Ki Hyoung Ko and Lawrence Smolinsky, A combinatorial matrix in 3-manifold theory

W. B. Raymond Lickorish, Invariants for 3-manifolds from the combinatorics of the Jones polynomial

Peter Arnold Linnell, Zero divisors and group von Neumann algebras . . . . . 349

Bruce Harvey Wagner, Classification of essential commutants of abelian

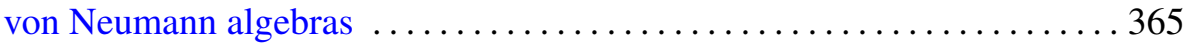

Herbert Walum, Multiplication formulae for periodic functions ..........383 